\title{
A quantitative study of the arrangement of the suprascapular nerve and vessels in the suprascapular notch region: new findings based on parametric analysis
}

\author{
H. Jezierski ${ }^{1}$, G. Wysiadecki², M. Sibiński ${ }^{3}$, A. Borowski ${ }^{3}$, M. Podgórski², M. Topol², \\ M. Polguj' \\ ${ }^{1}$ Department of Angiology, Interfaculty Chair of Anatomy and Histology, Medical University of Lodz, Poland \\ ${ }^{2}$ Department of Normal and Clinical Anatomy, Interfaculty Chair of Anatomy and Histology, \\ Medical University of Lodz, Poland \\ ${ }^{3}$ Clinic of Orthopaedic and Paediatric Orthopaedics, Medical University of Lodz, Poland
}

[Received: 22 December 2015; Accepted: 7 March 2016]

Background: When closed by the superior transverse scapular ligament (STSL), the suprascapular notch (SSN) creates an osseo-fibrous tunnel which acts as a pathway for the suprascapular nerve (SN). Anatomical variations are common in this region, and these can increase the risk of neuropathy by restricting the space for nerve passage. The aim of this study is to identify any correlation between the area reduction coefficient parameters and the $S N$ and vessel arrangements in the SSN region.

Material and methods: The SSN region was dissected in 88 formalin-fixed cadaveric shoulders (40 left and 48 right). During dissection, the topography of the SN, artery and vein was evaluated. Quantitative visual data analysis software was used to measure the areas of the STSL and the anterior coracoscapular ligament (ACSL), as well as the diameters of the SN and associated vessels, and to assign those structures to existing classifications. The area reduction coefficient (ARC) was calculated for each shoulder.

Results: The area of the STSL (aSTSL) and ACSL (aACSL) were significantly larger in Type IV than Type I of the triad. Similarly, the aSTSL and area of the SSN (aSSN) were found to be significantly larger in Type IV than Type III. However, no significant differences were found in the ARC of the STSL (ARC $S T S L$ ), the ARC of the ACSL $\left(A R C_{A C S L}\right)$ or the total ARC $\left(A R C_{\text {tota }}\right)$.

Conclusions: Although the aSTSL, aACSL and aSSN varied according to the type of SN and vessel arrangement, coefficient analysis ( $A R C_{S T S L^{\prime}} A R C_{A C S L}$ and $A R C_{\text {total }}$ ) indicated that combined effect of these variations did not significantly affect SSN morphology. (Folia Morphol 2016; 75, 4: 454-459)

Key words: anatomical variations, suprascapular nerve entrapment, suprascapular artery, suprascapular nerve, suprascapular vein

Address for correspondence: Assoc. Prof. M. Polguj, MD, PhD, Department of Angiology, Interfaculty Chair of Anatomy and Histology, Medical University of Lodz, ul. Narutowicza 60, 90-136 tódź, Poland, tel: +48 42 6304949, e-mail: michal.polguj@umed.lodz.pl 


\section{INTRODUCTION}

The most common point for suprascapular nerve (SN) compression and traction injury is the suprascapular notch (SSN) $[9,23]$. Suprascapular neuropathy is characterised by weakness of the arm and by chronic, diffuse pain in the posterior and/or lateral region of the shoulder, which may radiate down the forearm or up into the ipsilateral neck region $[3,23]$. This pathology is also important from a demographic point of view because it mainly occurs in patients under 35 years of age [1, 23].

The natural passage of the SN through the SSN is constant in that it always passes under the superior transverse scapular ligament (STSL) [18, 21, 22]. However, the locations of the associated suprascapular vein and artery are highly variable [16, 20, 22].

Previous studies of the aetiopathology of SN entrapment have paid little attention to the topography of the suprascapular triad: the nerve, artery and vein. Nevertheless, this is a very important subject, because the position of the suprascapular artery under the STSL could be one of the possible causes of SN entrapment $[2,16,19,20]$.

This article offers a detailed study of the correlation between the topography of the suprascapular triad (nerve, artery and vein) and reductions in the area of the SN passage at the SSN region. A better understanding of these parameters may improve the quantitative evaluation of the risk of SN neuropathy in different arrangements of the suprascapular triad at SSN. In contrast to previous studies, the present study presents a precise description of the area reduction by the ligament at the SSN made possible by its use of image analysis software. Earlier observations of the suprascapular triad have been only macroscopic and approximate, and not supported by such complex calculations.

\section{MATERIALS AND METHODS}

Eighty-eight formalin-fixed, cadaveric shoulders from a Polish population (40 left and 48 right) were dissected. The sex and age of the donors were unknown. Ethical approval to undertake this study was obtained from the Bioethics Commission of the Medical University of Lodz (protocol no. RNN/12//10/KE). In all shoulders, a horizontal incision along the clavicle was performed. Next, the skin was separated from the trapezius, deltoid and pectoralis major muscles. The trapezius and the deltoid muscles were then disinserted and reclined to expose the supraspinatus, infraspinatus and subscapularis muscles. After retraction of the supraspinatus muscle from the supraspinatus fossa, the superior border of the scapula and superior transverse scapular ligament were visualised. The morphological characteristics of the structures in the SSN region were noted, as were the relationships between the $\mathrm{SN}$, artery and vein with any other structures and the presentation of any abnormal masses in this area. The arrangement of the suprascapular nerve and vessels was identified in relation to the superior transverse suprascapular ligament.

In all specimens, photographic documentation of structures in the SSN region was obtained. The arrangement of the suprascapular nerve and vessels was classified according to the most recent classification [16]. The areas of the SN passage, STSL and ACSL (when the ligament was present) were measured by MultiScanBase 18.03 quantitative image analysis software (Computer Scanning System II, Warsaw, Poland) as described previously [12, 15].

Based on the analysis of the photographic documentation, the area reduction coefficient (ARC) was calculated according to the following formula [11] (Fig. 1):

$$
\mathrm{ARC}_{\text {total }}[\%]=\mathrm{ARC}_{\mathrm{STSL}}[\%]+\mathrm{ARC}_{\mathrm{ACSL}}[\%],
$$

when ACSL present area reduction coefficient of the STSL $\left(\right.$ ARC $_{\text {STSL }}$ ) (Fig. 1A):

$$
\mathrm{ARC}_{\mathrm{STSL}}[\%]=(\mathrm{aSTSL} / \mathrm{aSSN}) \times 100 ;
$$

area reduction coefficient of the $A C S L\left(A_{A C S L}\right)$ (Fig. 1B):

$$
\mathrm{ARC}_{\mathrm{ACSL}}[\%]=(\mathrm{aACSL} / \mathrm{aSSN}) \times 100 ;
$$

when: aSSN (area of the suprascapular notch) - the area limited by the superior border of the STSL at the line of proximal and distal attachment and the bony border of the SSN; aSTSL (area of the superior transverse scapular ligament) - the area limited by the superior and inferior border of the STSL and the bony borders of the SSN; aACSL (area of the anterior coracoscapular ligament) - the area limited by the superior and inferior border of the ACSL and the bony borders of the SSN; aSSO (area of the suprascapular opening) - the area of the suprascapular notch that is not occupied by the STSL or the ACSL. When the ACSL is absent, the aSSO is identical with the area of the SN passage (Fig. 1A); however, when the ACSL is present, it divides the aSSO into the SN passage area and the opening between its lower border and the bony border of the SSN (Fig. 1B).

The topography of the suprascapular nerve and vessels was determined based on the fourfold classification defined by Polguj et al. [16]. The classification is as follows: Type I, where the suprascapular artery runs above the ligament and the suprascapular vein 


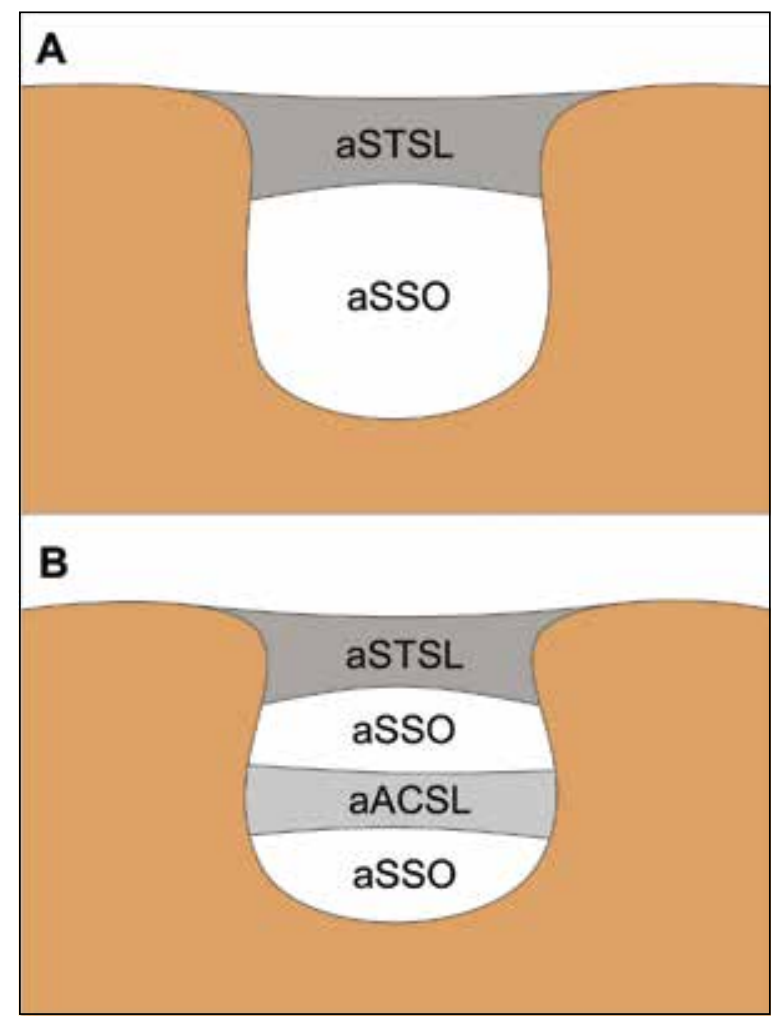

Figure 1. Schematic representation of the measurements of the areas in the suprascapular region - area of the superior transverse scapular ligament (aSTSL); area of the anterior coracoscapular ligament (aACSL); area of the suprascapular opening (aSSO); A. Specimens without ACSL; B. Specimens with ACSL.

and nerve below it; Type II, where both vessels pass above the ligament and the nerve passes under it; Type III, where the suprascapular vessels and nerve lie under the ligament; and Type IV, which comprises the other variants of these structures (Figs. 2-5).

\section{Statistical analysis}

Statistica 12.0 software (StatSoft Polska, Krakow, Poland) was employed for all statistical analyses. The normality of data distribution was checked with the Shapiro-Wilk test. The Kruskal-Wallis one way ANOVA, with dedicated post hoc tests, was used to compare the specimens from four different groups. A p-value $<0.05$ was concerned significant.

\section{RESULTS}

In all specimens, the SN travelled below the superior transverse scapular ligament. The mean diameter of the SN was found to be $2.4 \pm 0.33 \mathrm{~mm}$ (mean \pm \pm standard deviation). The mean diameters of the suprascapular artery and vein were $2.16 \pm 0.51 \mathrm{~mm}$ and $3.18 \pm 0.55 \mathrm{~mm}$, respectively.

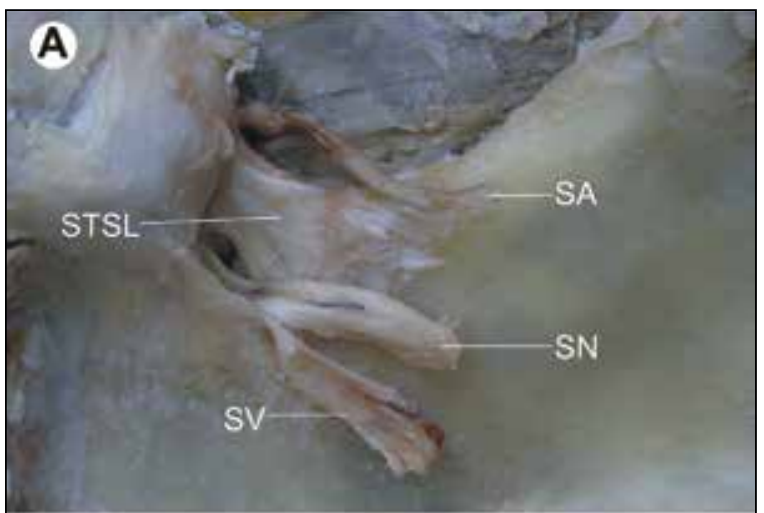

(B)

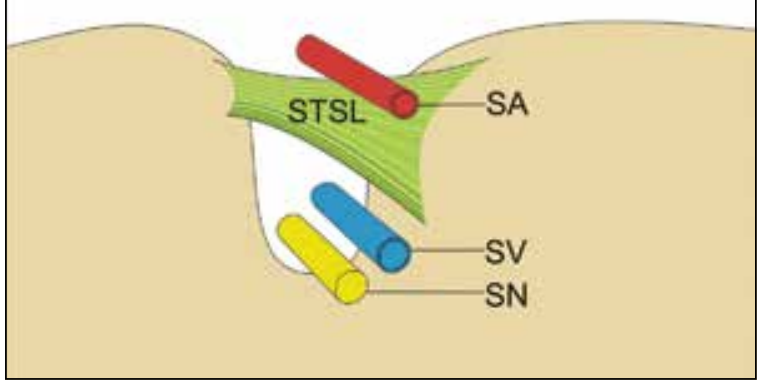

Figure 2. Type I arrangement of the suprascapular nerve (SN), suprascapular artery (SA) and suprascapular vein (SV) at the suprascapular notch; A. Structures at the cadaver; B. Schematic arrangements; STSL — superior transverse scapular ligament.

\section{Distributions of the suprascapular nerve and vessel arrangements}

The distribution of the arrangements of the suprascapular vein, artery and nerve according to the classification given above in the region of the SSN was as follows: Type I (52/88; 59.1\%) (Fig. 2), Type II (17/88; 19.3\%) (Fig. 3), Type III (10/88; 11.4 \%) (Fig. 4) and Type IV (9/88; 10.2\%) (Fig. 5).

\section{Comparison of the surface area parameters (aSTSL, aACSL, aSSN)}

The mean surface areas of the STSL (aSTSL) in the specimens according to suprascapular triad type were $55.12 \mathrm{~mm}^{2}$ (Type I), $58.02 \mathrm{~mm}^{2}$ (Type II), $47.01 \mathrm{~mm}^{2}$ (Type III) and $77.35 \mathrm{~mm}^{2}$ (Type IV). According to the ANOVA these differences were significant and the post hoc test indicated that it was due to significantly larger aSTSL in Type IV than inTypes I and III (Table 1).

The mean surface areas of the ACSL (aACSL) was greater in Type IV specimens $(14.41 \mathrm{~mm})$ than Type I $(4.74 \mathrm{~mm})$, Type II $(6.00 \mathrm{~mm})$ and Type III specimens $(4.74 \mathrm{~mm})$. The post hoc tests found that the aACSL was significantly larger in Type IV than Type I (Table 1). 


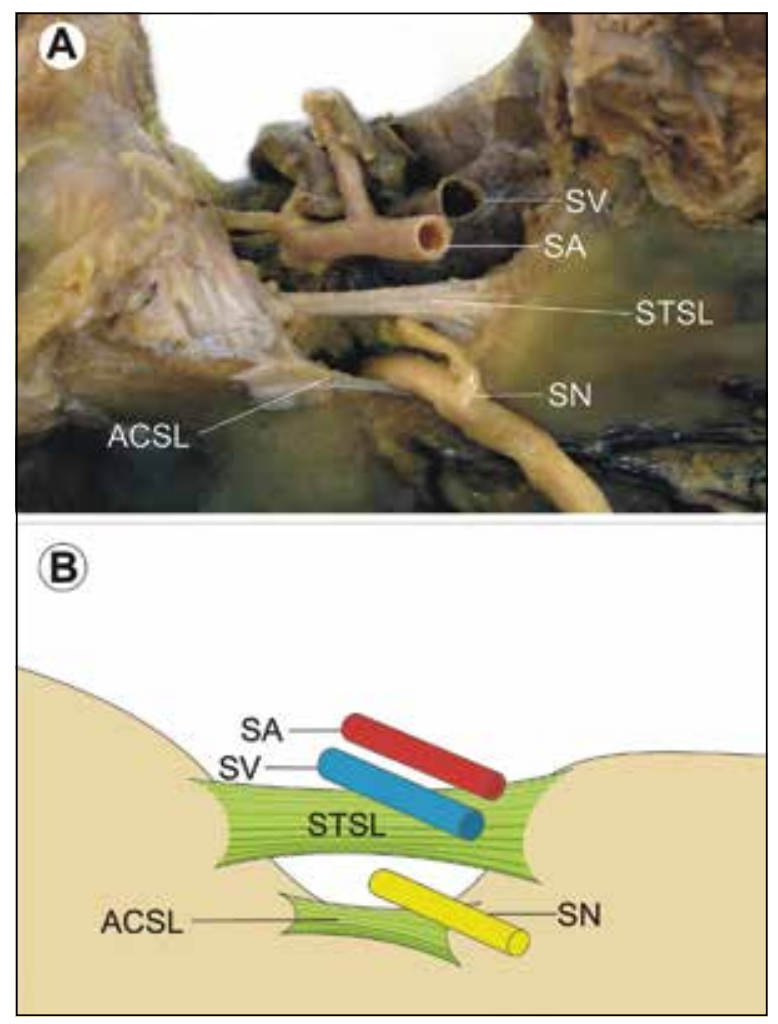

Figure 3. Type II arrangement of the suprascapular nerve (SN), suprascapular artery (SA) and suprascapular vein (SV) at the suprascapular notch; A. Structures at the cadaver; B. Schematic arrangements; ACSL — anterior coracoscapular ligament; STSL — superior transverse scapular ligament.

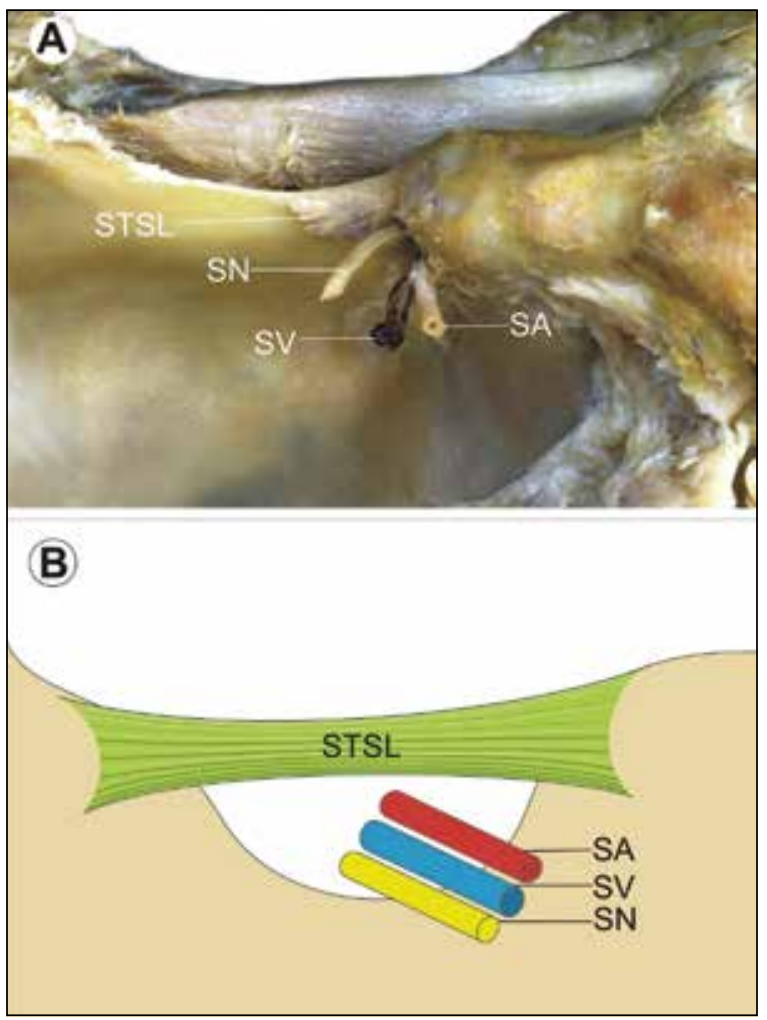

Figure 4. Type III arrangement of the suprascapular nerve (SN), suprascapular artery (SA) and suprascapular vein (SV) at the suprascapular notch; A. Structures at the cadaver; B. Schematic arrangements; STSL — superior transverse scapular ligament.

Table 1. Comparison between area reduction coefficients, areas of ligaments and areas of the suprascapular notch between different types of triad

\begin{tabular}{|c|c|c|c|c|c|}
\hline \multirow[t]{2}{*}{ Parameter } & \multicolumn{4}{|c|}{ Type of triad } & \multirow[t]{2}{*}{$\mathbf{P}$} \\
\hline & I & II & III & IV & \\
\hline aSTSL [mm²] & $55.12(21.66)$ & $58.02(24.48)$ & 47.01 (21.12) & $77.35(18.10)$ & 0.0313 \\
\hline aACSL [mm²] & $4.74(6.87)$ & $6.00(7.53)$ & $4.74(4.99)$ & $14.41(9.69)$ & 0.0354 \\
\hline aSSN & $94.40(35.40)$ & 93.65 (29.42) & $73.51(27.52)$ & $131.61(43.95)$ & 0.0372 \\
\hline $\mathrm{ARC}_{\mathrm{STSL}}[\%]$ & $59.46(12.23)$ & $61.04(9.47)$ & $62.45(13.93)$ & $60.67(8.88)$ & 0.8327 \\
\hline $\mathrm{ARC}_{\mathrm{ACSL}}[\%]$ & $5.09(7.48)$ & $7.30(9.15)$ & $6.19(6.09)$ & $10.32(4.91)$ & 0.1111 \\
\hline ARC [\%] & 64.55 (11.34) & $68.34(12.27)$ & 68.64 (12.99) & 70.99 (9.87) & 0.2275 \\
\hline
\end{tabular}

Abbreviations - see text

The area of the suprascapular notch (aSSN) was the greatest in Type IV $\left(131.61 \mathrm{~mm}^{2}\right)$ and lowest in Type III $\left(73.51 \mathrm{~mm}^{2}\right)$. Also the aSSN was significantly larger in Type IV than Type III (Table 1).

\section{Area reduction coefficient (ARC)}

No statistically significant difference was found between the four groups with regard to the ARC values of the STSL (ARC STSL $_{\text {) }}$ ) 59.46\% (Type I), $61.04 \%$ (Type II), 62.45\% (Type III), and 60.67\% (Type IV) (Table 1).

The mean area reduction coefficient of the ACSL $\left(\mathrm{ARC}_{\mathrm{ACSL}}\right)$ of Type IV (10.32\%) was significantly greater than that of Type I (5.09\%), Type II (7.30\%) or Type III (6.19\%). However, no other significant differences were observed (Table 1). 


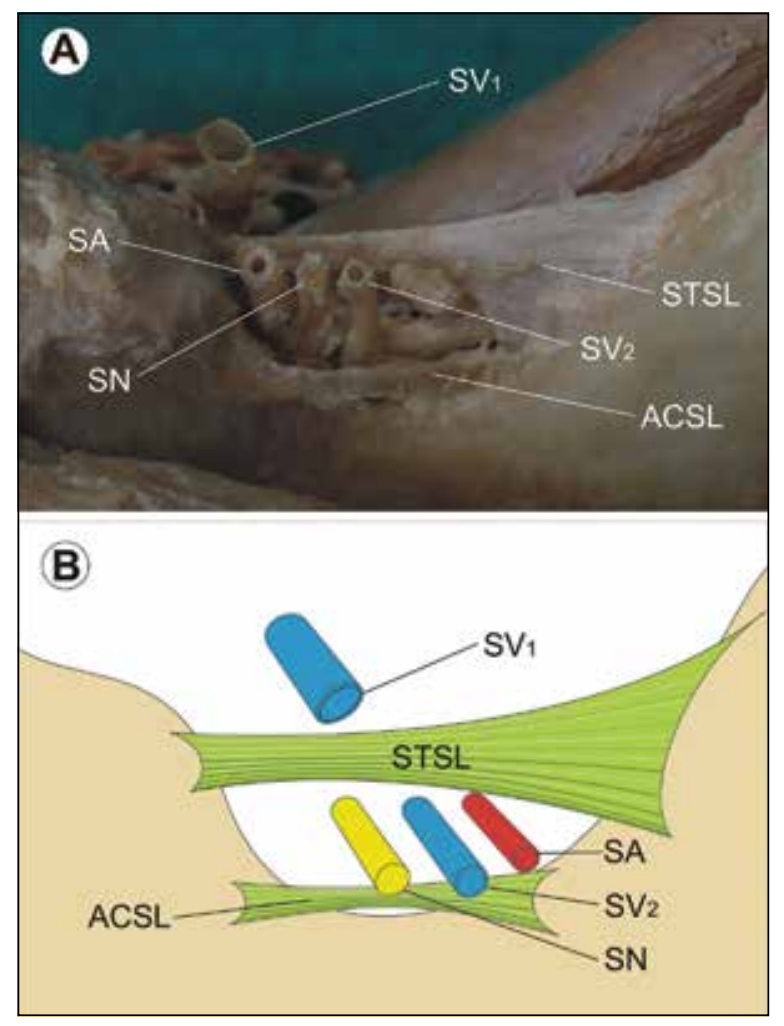

Figure 5. Type IV arrangement of the suprascapular nerve (SN), suprascapular artery (SA) and suprascapular vein (SV) at the suprascapular notch; A. Structures at the cadaver; B. Schematic arrangements; SV 1 - first SV; SV 2 - second SV; ACSL — anterior coracoscapular ligament; STSL — superior transverse scapular ligament.

The mean total area reduction coefficients $\left(\mathrm{ARC}_{\text {total }}\right.$ ) were quite similar for Types I-III: 64.55\% (Type I), $68.34 \%$ (Type II), $68.64 \%$ (Type III). The value was higher for Type IV (70.99\%). However, again, no other statistically significant differences were observed (Table 1).

\section{DISCUSSION}

A current bibliography search reveals only two classifications of the suprascapular vein, artery and nerve location at SSN (by Yang et al. [22], and by Polguj et al. [16]). The present study uses the fourfold, classification defined by Polguj et al. [16] as it is clearer and more practical in clinical practice.

Yang et al. [22] distinguish three types of suprascapular vessel arrangement based on an examination of 103 shoulders. In Type I (60 shoulders; 59.4\%), all suprascapular vessels run over the STSL; in Type II (30 shoulders; $29.7 \%$ ), the vessels run over and under the STSL simultaneously; in Type III (11 shoulders; $10.9 \%$ ), all vessels run under the STSL.

In another study, Yang et al. [22] divide the Type II arrangement into four subtypes. In Type IIA (12 shoulders), the suprascapular artery and vein cross over the STSL and another vein runs under the STSL. However, in two Type IIA shoulders, double suprascapular veins crossed over the STSL and another vein ran under the STSL. In Type IIB (4 shoulders), the suprascapular artery and vein run under the STSL, and another vein crosses over the STSL. In Type IIC (3 shoulders), the suprascapular artery crosses over the STSL while the vein runs under the STSL. In Type IID (11 shoulders), the suprascapular artery runs under the STSL while the vein crosses over the STSL.

Our results found that the most common arrangement of the suprascapular triad at the SSN was Type I, with the suprascapular artery running above the ligament and the suprascapular vein and nerve running below it (52 shoulders; $59.1 \%$ ). Although this arrangement is comparable with type IIC according to Yang et al. [22], the rates of occurrence are significantly different, with only three of our shoulders meeting Yang's IIC criterion. Also, although Yang's Type I configuration and our Type II are similar, i.e. both vessels pass above the ligament with the nerve passing under it, the frequency of occurrence was found to be considerably higher (59.4\%) in Yang et al. [22] than the present study (17\%). The frequency of Type III in our study (12.3\%) was found to be similar to Yang et al. [22] (10.9\%). It may be that such significant differences in frequency of location of the suprascapular nerve and vessels at the SSN depend on the studied population. It would be reasonable to assume that its occurrence varies worldwide based on population.

Our description of the SN course in the SSN is similar to those given by Bayramoglu et al. [2], Greiner et al. [6], Polguj et al. [16] and Piyawinijwong and Tantipoon [10], where it was always found to travel below the superior transverse scapular ligament. The diameter of the SN measured in the present study is $2.4 \pm 0.33 \mathrm{~mm}$. This value is similar to previous descriptions: $2 \mathrm{~mm}$ [7], $2.5 \mathrm{~mm}$ [22], $3 \mathrm{~mm}[5,8]$.

The large number of anatomical variations located at the SSN makes it a very heterogeneous region. The shapes of the SSN and the STSL or ACL are highly diverse $[2,12-14,18]$. A tight and narrow SSN or a broad, bifid or ossified STSL can all obstruct the passage of the $\mathrm{SN}$, increasing the risk of its injury, and predisposing the patient to the development of SN neuropathy $[2,23]$. In addition, the presence of an artery in the osteo-fibrous tunnel that usually houses only the nerve and vein can also reduce the amount of available area. Such anomalies of artery alignment were observed in $11.4 \%$ of analysed cases. This rate is higher than the $2.5 \%$ reported by Tubbs 
et al. [20] and 3\% by Reineck et al. [17], but similar to the $10.9 \%$ reported by Yang et al. [22]. It was suggested that when the artery directly neighbours the nerve, the pressure it exerts may cause microtrauma to the more fragile nerve, eventually resulting in neuropathy [20]. Also, Ringel et al. [19] postulate that vascular microtrauma may cause nerve dysfunction. However, due to the difficulties concerned with the clinical evaluation of those small vessels and their unconfirmed aetiological status, this was not taken into consideration in the present study.

Chen and Adds [4] report the presence of an accessory suprascapular artery which accompanied the $\mathrm{SN}$ under the superior transverse scapular ligament. Although no such vessels were found in the present study, this is an important finding because they may well reduce space for SN passage and contribute to the supply of the middle third of the clavicle by several periosteal branches.

The results of this paper may also have clinical value. The topography of the suprascapular triad should be regarded as an important risk factor during endoscopic and open SN decompression for suprascapular vein and artery injury, particularly in the case of arrangements III and IV.

\section{CONCLUSIONS}

Although the surface areas of the STSL, ACSL and SSN (aSTSL, aACSL and aSSN) differed between the four types of suprascapular nerve and vessel arrangement at the SSN, the analysis of their coefficients $\left(A_{R C} C_{\text {STS }}, A R C_{A C S L}\right.$ and $A R C_{\text {total }}$ ) indicated that the cumulative effect of these variations did not significantly affect the morphology of the SSN region.

\section{REFERENCES}

1. Antoniadis G, Richter HP, Rath S, Braun V, Moese G (1996) Suprascapular nerve entrapment: experience with 28 cases. J Neurosurg. 85: 1020-1025.

2. Bayramoglu A, Demiryurek D, Tuccar E, Erbil M, Aldur MM, Tetik O, Doral MN (2003) Variations in anatomy at the suprascapular notch possibly causing suprascapular nerve entrapment: an anatomical study. Knee Surg Sport Trum Arthrosc, 11: 393-398.

3. Boykin RE, Friedman DJ, Zimmer ZR, Oaklander AL, Higgins LD, Warner JJ (2011) Suprascapular neuropathy in a shoulder referral practice. J Shoulder Elbow Surg, 20: 983-988.

4. Chen D, Adds $P$ (2011) Accessory suprascapular artery. Clin Anat, 24: 498-500.

5. Goubier JN, Teboul F (2015) Transfer of the rhomboid nerve to the suprascapular nerve: An anatomical feasibility study. Chir Main, 34: 182-185.

6. Greiner A, Golser K, Wambacher M, Kralinger F, Sperner G (2003) The course of the suprascapular nerve in the supraspinatus fossa and its vulnerability in muscle advancement. J Shoulder Elbow Surg, 12: 256-259.
7. Harmon D, Hearty C (2007) Ultrasound-guided suprascapular nerve block technique. Pain Physician, 10: 743-746.

8. Hirokawa D, Eliezri Y, Desciak E, Campanelli C (2010) Suprascapular nerve injury during Mohs surgery and review of the surgical anatomy of the nervous structures of the supraclavicular triangle. Dermatol Surg, 36: 1756-1758.

9. Lee JJ, Yoo YS, Hwang JT, Kim DY, Jeon SJ, Hwang SM, Jang JS (2015) Efficacy of direct arthroscopy-guided suprascapular nerve block after arthroscopic rotator cuff repair: a prospective randomized study. Knee Surg Sports Traumatol Arthrosc, 23: $562-566$

10. Piyawinijwong S, Tantipoon P (2012) The anterior coracoscapular ligament in Thais: possible etiological factor of suprascapular nerve entrapment. Siriraj Med J, 64: S12-S14.

11. Podgórski M, Topol M, Sibiński M, Grzelak P, Stefańczyk L, Polguj M (2014) New parameters describing morphological variations in the suprascapular notch region as potential predictors of suprascapular nerve entrapment. BMC Musc Disord, 15: 396.

12. Polguj M, Jędrzejewski K, Majos A, Topol M (2012) Variations in bifid superior transverse scapular ligament as a possible factor of suprascapular entrapment: an anatomic study. Int Orthopaed, 36: 2095-2100.

13. Polguj $M$, Jędrzejewski $K$, Podgórski M. Topol M (2011) Correlation between morphometry of the suprascapular notch and antropometrical measurments of the scapula. Folia Morphol, 70: 109-115.

14. Polguj M, Jędrzejewski K, Topol M (2013) Variable morphology of the anterior coracoscapular ligament: a proposal of classification. Ann Anat, 195: 77-81.

15. Polguj M, Podgórski M, Jędrzejewski K, Topol M (2012) The double suprascapular foramen: unique anatomical variation and the new hypothesis of its formation. Skel Rad, 41: 1631-1636.

16. Polguj M, Rożniecki J, Sibiński M, Grzegorzewski A, Majos $A$, Topol M (2015) The variable morphology of suprascapular nerve and vessels at the suprascapular notch: a proposal for classification and its potential clinical implications Knee Surg Sports Traumatol Arthrosc, 23: 1542-1548.

17. Reineck JR, Krishnan SG (2009) Subligamentous suprascapular artery encountered during arthroscopic suprascapular nerve release: a report of three cases. J Shoulder Elbow Surg, 18: e1-3.

18. Rengachary SS, Burr D, Lucas S, Hassanein KM, Mohn MP, Matzke H (1979) Suprascapular entrapment neuropathy: a clinical, anatomical, and comparative study. Part 2: anatomical study. Neurosurgery, 5:447-451.

19. Ringel SP, Treihaft M, Carry M, Fisher R, Jacobs $P(1990)$ Suprascapular neuropathy in pitchers. Am J Sports Med, 18: 80-86.

20. Tubbs RS, Smyth MD, Salter G, Oakes WJ (2003) Anomalous traversement of the suprascapular artery through the suprascapular notch: a possible mechanism for undiagnosed shoulder pain? Med Sci Monit, 9: BR116-BR119.

21. Urguden M, Ozdemir H, Donmez B, Bilbasar H, Oguz N (2004) Is there any effect of suprascapular notch type in iatrogenic suprascapular nerve lesions? An anatomical study. Knee Surg Sports Traumatol Arthrosc, 12: 241-245.

22. Yang HJ, Gil YC, Jin JD, Ahn SV, Lee HY (2012) Topographical anatomy of the suprascapular nerve and vessels at the suprascapular notch. Clin Anat, 25: 359-365.

23. Zehetgruber $H$, Noske $H$, Lang T, Wurnig C (2002) Suprascapular nerve entrapment: a meta-analysis. Int Orthop, 26 : 339-343. 